\title{
Medizinisches Wissen in der Unfallversicherung
}

\author{
Die Begutachtung von „Preßluftschäden“ im Krankenhaus \\ Bergmannsheil, 1929-1944
}

\section{Alexa Geisthövel}

Medical Knowledge in Accident Insurance. Assessing "Pneumatic Tool Damages" at the Bergmannsheil Hospital, 1929-1944

\begin{abstract}
The article focuses on one central element of medical activity in the context of the German social insurance system: providing expert assessments in accident pension cases. Taking an example from interwar coal mining, it aims to reconstruct how social policy makers first conceived of "pneumatic tool damages" as occupational disease and how trauma surgeons had to deal with this new entity of social law once it had been institutionalized in 1929. Drawing on physicians' publications as well as archival sources from the supreme court in social insurance, the Reichsversicherungsamt, the article examines how the controversial generation of new knowledge took place. It argues that medical knowledge was neither simply applied to administration and law nor was it compromised by the necessity to adjust it to those fields of decision-making. Expert medical opinions should instead be understood as a specific form of medical knowledge.
\end{abstract}

Der Beitrag nimmt eine zentrale ärztliche Tätigkeit im Rahmen des deutschen Sozialversicherungswesens in den Blick: die Begutachtung in Unfallrentenangelegenheiten. Am Beispiel des Kohlenbergbaus in der Zwischenkriegszeit rekonstruiert er, wie zuerst Sozialpolitiker den „Preßluftschaden“ als Berufskrankheit definierten, woraufhin Unfallchirurgen diesem sozialrechtlichen Tatbestand eine ärztliche Praxis abgewinnen mussten. Auf Grundlage medizinischer Fachpublikationen und der Archivalien des höchsten Spruchgremiums im Sozialrecht, des Reichsversicherungsamtes, wird die kontroverse Hervorbringung von neuem Wissen nachgezeichnet. Der Beitrag argumentiert, dass medizinisches Wissen in der Begutachtung weder einfach „angewandt" noch dass es kompromittiert wurde, wenn sich Ärzte an den Rationalitäten von Verwaltung und Rechtsprechung orientierten. Medizinische Begutachtung sollte stattdessen als eine spezifische Form des ärztlichen Wissens verstanden werden.

Das deutsche Sozialversicherungswesen gilt als eines der Felder, in denen Mediziner bereits seit dem letzten Drittel des 19. Jahrhunderts das "Soziale" durch die Bereitstellung von wissenschaftlichem Wissen mitregierten (Raphael 1996: 168). Davon zeugt unter anderem eine umfangreiche historiographische Literatur über die Bestimmung, Regulierung und 
Verwaltung von Arbeitsunfällen oder Berufskrankheiten. Allerdings stehen hier die Aushandlungsprozesse zwischen verschiedenen polit-ökonomischen Gruppen von Akteuren - Arbeitgeber und Arbeitnehmer, Versicherungsträger, Sozialpolitiker in Regierungen und Parlamenten sowie Politikberater aus verschiedenen Wissenschafts- und Praxiszweigen - im Vordergrund. Die epistemischen Dynamiken der Sozialversicherung haben demgegenüber bisher deutlich weniger Beachtung gefunden. Als sehr aufschlussreich hat es sich erwiesen, die Regierung des „Risikos“ bzw. der „Produktivität“ im Sinne eines modernen biopolitischen Paradigmas mit der verpflichtenden Gesundheitssicherung seit dem späten 19. Jahrhundert in Verbindung zu bringen. ${ }^{1}$ Dabei wurde nicht nur gezeigt, wie die Verrechtlichung und Verwissenschaftlichung industrieller Arbeit und ihrer pathogenen Folgen neues Expertenwissen aus unterschiedlichen Disziplinen mobilisierte (Moses 2012: 60), sondern auch die Etablierung bestimmter „styles of reasoning“ (Ian Hacking), etwa des stochastisch-statistischen, beschrieben. Die Kehrseite einer solch theoretisch stringenten Perspektivierung ist, dass sie das Problem der medizinischen Wissensbildung in der Sozialversicherung vorschnell vereindeutigt, statt es weiter $\mathrm{zu}$ öffnen und erst einmal alle beteiligten, womöglich sehr heterogenen Praktiken (Lengwiler 2017) genauer zu untersuchen.

Ein zentrales ärztliches Tätigkeitsfeld in der sozialen Sicherung, bei dem ein solches Unterfangen ansetzen könnte, war die medizinische Begutachtung im Rahmen von Unfallrentenverfahren (Knoll-Jung 2017). Diese "Gutachtenmedizin“ ist in der sozialhistorischen Literatur teils als Komplizenschaft williger Ärzte mit Arbeitgebern und konservativen Sozialpolitikern, teils als den Ärzten aufgezwungene Entpolitisierung ihres medizinischen Handelns für Menschen in Not beschrieben worden (etwa Milles 1999: 31-32). Ich möchte hingegen dafür plädieren, Begutachtung nicht als instrumentalisierte Schwundstufe der Medizin, sondern als eine spezifische Form der medizinischen Wissensgenerierung zu verstehen, die an der Schnittstelle von Klinik, Versicherung und Rechtsprechung unter noch zu klärenden Bedingungen stattfand.

Beispielsweise führten massenhafte Begutachtungen Ärzten ein sonst nicht verfügbares „Beobachtungsmaterial“ zu (vgl. etwa Martin 2014: 229) und forderten laufend Entscheidungen in Fragen ein, auf die Mediziner noch keine Antworten gefunden hatten. Wie die Anleitungsliteratur zeigt, gründete sich Gutachtenwissen auf eigene Routinen, medizinisches Wissen konnte in Begutachtungen nicht einfach "angewendet“ werden, weil hierbei stets verschiedene Wissensfelder (menschlicher Körper/Arbeitsmarkt/ Sozialrecht und andere) miteinander verschränkt waren. Nicht zuletzt war medizinische Begutachtung nicht zwingend mit dem Anspruch verknüpft, wissenschaftlich „objektive“ Aussagen zu machen. Eine Rhetorik der Objek- 
tivität gab es von Seiten der Ärzte wie ihrer Auftraggeber, jedoch zeigt sich bei genauerem Hinsehen, dass gerade erfahrene Gutachter ausdrücklich zwischen gerechtfertigten Gutachterurteilen auf der Grundlage unsicheren Wissens und dem Konsens der scientific community in Bezug auf einen bestimmten Sachverhalt unterschieden (Geisthövel \& Hess 2017: 21-25).

Um das Forschungsproblem "Gutachtenwissen“ an einem konkreten Beispiel zu umreißen, bietet sich das soziale Sicherungswesen im Bergbau an, da dieses vergleichsweise gut erforscht ist und einige neuere Arbeiten bereits auf die Begutachtung von bergmännischen Leiden eingehen, wenngleich die beteiligten Praktiken dabei nur selektiv verhandelt werden (zuletzt Martin 2014; Bluma 2015). Mein Beispiel beschäftigt sich mit einem kleinen, unspektakulären Wissenszuwachs im Gebiet der Unfallchirurgie (Schlich 2006), die als medizinisches Fach eng mit der gesetzlichen Unfallversicherung verwoben war. Ausgangspunkt ist eine sozialpolitische Setzung, die in den 1920er Jahren das bestehende System der betrieblichen Unfallversicherung modifizierte, indem sie eine Reihe von „Berufskrankheiten" ebenfalls entschädigungspflichtig machte. Unter diesen ragen die „Schädigungen durch Arbeit mit Preßluftwerkzeugen“ nicht sonderlich hervor. Sie waren weder weit verbreitet noch tödlich, weshalb es sich um ein relativ überschaubares Problem handelt, dessen Entfaltung sich vom Erlass der entsprechenden Verordnung über die medizinische Eingrenzung und Stabilisierung des administrablen und justiziablen Sachverhalts bis zu weiteren wissenschaftlichen und gesetzgeberischen Anschlusshandlungen verfolgen lässt. Pressluftschäden sind auch deshalb interessant, weil sie etwa im Gegensatz zu den Staublungenerkrankungen unter Medizinern gar nicht als Berufskrankheit diskutiert worden waren, bis die Gesetzgeber sie $\mathrm{zu}$ einer solchen erklärten. Medizinisches Nichtwissen stand geradezu am Beginn dieses sozialrechtlichen Faktums, wobei sich eine einzelne klinische Einheit schnell eine Art Begutachtungsmonopol erwarb: die chirurgische Abteilung am Krankenhaus Bergmannsheil in Bochum, das ebenfalls ein Zentrum der Silikosebegutachtung und -forschung war (Martin 2002; Martin 2014).

\section{Kontaktzone Unfallkrankenhaus}

Seit 1890 betrieb die II. Sektion der Knappschafts-Berufsgenossenschaft - Trägerin der gesetzlichen Unfallversicherung im Ruhrkohlenbergbau in Bochum ein eigenes Unfallkrankenhaus. Von Anfang an bildete das Bergmannsheil eine markante "wissenschaftlich-technische Verdichtung" in der Krankenhauslandschaft des Ruhrgebiets (Bluma 2010: 76; vgl. auch 
Ostermann 1976: 28-29, 32), die in den folgenden Jahrzehnten räumlich, personell und technisch ständig ausgebaut wurde. Dies rechnete sich aus Sicht der Berufsgenossenschaft, weil sich Rentenzahlungen vermeiden ließen, wenn ein Unfallhergang sofort rekonstruiert, der Verunfallte effektiv behandelt und der stets befürchteten „neurotischen“ Fixierung entgegengearbeitet werden konnte.

In diesem Zusammenhang von Kontrolle, Kostenersparnis und moderner Behandlung wurde das Bergmannsheil zu einem Zentrum für die Begutachtung aller Unfallrentenempfänger der Knappschaft (Ostermann 1976: 27). In der zweiten Hälfte der 1920er Jahre stieg die Zahl der Begutachtungen, die auf der chirurgischen Abteilung für die Sektion II vorgenommen wurden, sprunghaft an, von gut 4.800 im Jahr 1926 auf knapp $6.200 \mathrm{im}$ Jahr 1927. Fast 11.000 Gutachten wurden auf dem Höhepunkt 1931 erstellt, danach sank die Zahl wieder ab bis auf gut 6.100 im Jahr 1933 (Ostermann 1976: 48, 52). In den Kriegsjahren zwischen 1939 und 1942 halbierte sich die Gutachtertätigkeit noch einmal (Boyer 1990: 60). ${ }^{2}$

Die am Bergmannsheil tätigen Ärzte waren keine Vertrauensärzte der Berufsgenossenschaft. „Medizin im Dienste der Unfallversicherung“ (Lohmar 1929: 552) betrieben sie aber insofern, als Begutachtung in ihrer Arbeitspraxis den gleichen Rang wie die Behandlung einnahm. Das Bergmannsheil bildete eine Schnittstelle zwischen administrativ-juristischer Handhabung des Sozialrechts und medizinischer Wissenschaft. Einerseits war es ein Institut des Unfallversicherungsträgers, der zugleich die Arbeitgeber der Versicherten organisierte, andererseits eine Fachklinik, die sich wissenschaftliches Renommee erarbeitete, im Fall der Chirurgie unter den Chefärzten Georg Magnus (1925-1933) und Heinrich Bürkle de la Camp (1933-1962).

Diese Doppelfunktion erlaubte es den dort arbeitenden Ärzten, Kontaktzonen für Mediziner, Funktionäre von Berufsgenossenschaften und Privatversicherungen, technische Beamte der Bergbauunternehmen sowie Juristen der Spruchbehörden herzustellen. Das geschah zum einen durch Publikationstätigkeit sowohl in Organen der Berufsgenossenschaft wie dem Kompaß - der häufig Entscheide des Reichsversorgungsamtes mit Auszügen der Bergmannsheil-Gutachten abdruckte -, als auch in Fachzeitschriften. Persönlich traf man sich auf unfallmedizinischen Fortbildungskursen, in einer unfallmedizinischen Arbeitsgemeinschaft (Lohmar 1929) oder auf der VIII. Tagung der Deutschen Gesellschaft für Unfallheilkunde, Versicherungs- und Versorgungsmedizin, die 1932 am Bergmannsheil stattfand.

Eine Kontaktzone bildete das Bergmannsheil zudem auf der praktischmaterialen Ebene der Falldokumentation. Das berufsgenossenschaftliche Krankenhaus ermöglichte es den Ärzten, auf die „mustergültig geführten Unfallakten“ (Scheffler 1927: 379) des Versicherungsträgers und damit auf 
medizinisch verwertbare Daten zuzugreifen, die sie selbst so nicht zu generieren vermochten. Denn um keine aus ihrer Sicht ungerechtfertigten Renten zahlen zu müssen, ließ die Berufsgenossenschaft betrieblich Verunfallte monatlich nachuntersuchen, bis die Verletzung ausgeheilt oder kein weiterer Heilungsfortschritt zu erwarten war. Diese engmaschige Beobachtungsroutine dokumentierte den Heilungsverlauf in einer Dichte, mit der es die Nachsorge im Krankenhaus nicht aufnehmen konnte.

Unfallmedizinische und allgemein-chirurgische Fragestellungen nutzten also Rentenzahlungs- und Erwerbsminderungsdaten, die einer ökonomischen und sozialrechtlichen Rationalität entsprangen. Umgekehrt profitierte der Versicherungsträger bzw. die Unfallversicherung als öffentlichrechtliche Institution von dieser ausschnitthaften Zweitverwertung sozialrechtlicher Daten, denn ihre Kernaufgabe war nicht die Wissensbildung, sondern die Erledigung administrativ-juristischer Vorgänge, die sich aus dem individuellen Rechtsanspruch der Versicherten ableiteten. Allerdings ergaben sich aus dieser Konstellation auch unvorhergesehene Effekte. So schuf die Berufsgenossenschaft unbeabsichtigt die Voraussetzung dafür, dass die Berufskrankheit „Staublunge“ in die Unfallversicherung aufgenommen wurde, indem sie im Bergmannsheil die Expertise zu deren Behandlung, Begutachtung und Erforschung konzentrierte (Boyer 1995: 131-135; Martin 2002: 103).

\section{Eine neue Berufskrankheit}

1925 dehnte eine Gesetzesänderung den betrieblichen Unfallschutz erstmals auf elf gewerbliche Berufskrankheiten aus. Am 11. Februar 1929 erweiterte eine Verordnung des Reichsarbeitsministers diese Liste um elf weitere Sachverhalte, darunter zwei, die für den Bergbau besonders relevant waren - neben den alles überragenden Staublungenerkrankungen auch die erste anerkannte mechanische Berufskrankheit (Thomann 1993): die „Erkrankungen der Knochen, Muskeln und Gelenke hervorgerufen durch die Arbeit mit Preßluftwerkzeugen".

Auf welches medizinische Wissen stützte sich die Einbeziehung dieser Leiden in den Versicherungsschutz? Lungenkrankheiten wurden schon lange mit dem Bergbau in Verbindung gebracht. In einigen Ländern waren bestimmte Varianten der "Staublunge“ seit dem frühen 20. Jahrhundert entschädigungspflichtig. Vielerorts gab es lokale Forschungen und in den 1920er Jahren eine wachsende grenzüberschreitende Zusammenarbeit von Arbeitsmedizinern und Sozialpolitikern, die die Anerkennung der „Silikose" als Berufskrankheit zu ihrem strategischen Ziel ausriefen (Lengwi- 
ler 2009; Rosental 2009). In diesem Zeitraum forschten sowohl Knappschaftsärzte als auch die Internisten am Bergmannsheil in diese Richtung und empfahlen, "schwere“ Formen von Staublungenerkrankungen in den Katalog der Berufskrankheiten aufzunehmen (Martin 2002: 104-105).

Anders sah es bei den Pressluftschäden aus. 1928 zitierte eine groß angelegte Studie über die Arbeitsverhältnisse im Steinkohlenbergbau die zuständigen Gewerbeärzte, im Bergbau sei keine Schädigung durch den Gebrauch von Pressluftwerkzeugen zu beobachten ([Lipmann] 1928: 227-228). Zwar hatte sich im Ruhrkohlenbergbau der Anteil der Presslufthämmer am Gewinnungsverfahren stark erhöht. Bis 1920 hatte er bei einigen wenigen Prozent gelegen, war danach angestiegen und verdoppelte sich zwischen 1925 und 1930 von rund 50 auf knapp 90 Prozent (Burghardt 1995: 290-293, Abb. 96, 291). Im Zusammenspiel mit der Einführung von Förderbändern und einer Konzentration der Betriebspunkte war diese rasante Verdrängung der Handarbeit durch Pressluftgeräte Teil der angestrebten „Rationalisierung“. Als potenziell gesundheitsgefährdend galt vor allem die Handhabung tragbarer Pressluftwerkzeuge mit Schlagwirkung (Abbauhammer) oder einer kombinierten Schlag- und Drehwirkung (Bohrhammer). Sie steigerten die Arbeitsproduktivität, reduzierten aber nicht den Kraftaufwand und belasteten Sehnen und Gelenke. Der Bergmann musste das bis zu 15 Kilogramm schwere, vibrierende Gerät nicht nur halten, sondern es sowohl anstemmen als auch den Rückstoß der bis zu 2.000 Schläge pro Minute abfangen (Wette 1931: 312-313; Rostock 1933: 89-91; Rostock 1934b: 634). Kasuistiken berichteten über Gesundheitsschäden am Bewegungsapparat, die durch Arbeit mit entsprechenden Geräten vor allem im metallverarbeitenden Gewerbe, in Steinbrüchen und im Straßenbau entstanden waren. Jedoch befielen diese nicht augenfällig einen Großteil der Bergleute. Vielmehr scheinen "Preßluftschäden“ präventiv und mehr oder weniger zufällig in die Versicherungspflicht aufgenommen worden zu sein, wie folgende Indizien nahelegen, die die fehlende archivalische Überlieferung ersetzen müssen.

1926 hatte der badische Gewerbeaufsichtsarzt Friedrich Holtzmann eine Kasuistik publiziert, die er im Rahmen eines Forschungsauftrags für das Reichsarbeitsministerium erhoben hatte. Dieser galt der Schwerhörigkeit von Kesselschmieden, die mit Pressluftgeräten arbeiteten. Als Nebenbefund stellte er bei einem Probanden verknöchernde Wucherungen und Abschleifungen am Schultergelenk fest und bezeichnete diese ebenfalls als „Berufserkrankung“ (Holtzmann 1926: 237). Zuständig für die Vorbereitung der erweiterten Berufskrankheitenliste war der Vorläufige Reichswirtschaftsrat. Dessen Arbeitsausschuss für die Reform der sozialen Versicherungsgesetze hörte am 8. Februar 1927 „ärztliche Sachverständige“ zu den beiden Themen Lärmschwerhörigkeit und Pressluftschäden - vermut- 
lich Holtzmann in Personalunion (1933: 132-133; vgl. auch Seyring 1930: 373). Der Ausschuss empfahl daraufhin, „[t]raumatische Erkrankungen der Knochen, Gelenke, Muskeln, Nerven und Blutgefäße, hervorgerufen durch Bedienung von Preßluftwerkzeugen“, in den Versicherungsschutz einzubeziehen.

Auch die Gewerkschafts-Zeitung, das Organ des Allgemeinen Deutschen Gewerkschaftsbundes (ADGB), verwies auf Holtzmanns Publikation. Sie zählte eine Fülle weiterer Beschwerden auf, die Arbeit an Pressluftgeräten zeitige, darunter „hochgradige Erschöpfung [...] des ganzen Körpers, Schlaflosigkeit, Gereiztheit, [...] Muskelzittern“ (Anonymus 1926: 672). Die Symptome entstammten einer Untersuchung im Auftrag des ADGB, die unter Beteiligung des Berliner Gewerbearztes Ernst Wilhelm Baader an einigen Berliner Arbeitern durchgeführt worden war ([Lipmann] 1928: 227). Solche Stichwörter waren geeignet, Neurosenalarm auszulösen. Im Verordnungsentwurf des Reichsarbeitsministeriums vom November 1928 war von Nerven und Gefäßen nicht mehr die Rede (Büro des vorläufigen Reichswirtschaftsrats 1933: 136-137). Ein Entscheid des Reichsversicherungsamts erläuterte später, Nervenerkrankungen seien nicht erwähnt worden, um keine falschen Entschädigungsansprüche bei funktionellen und zentralnervösen Erkrankungen zu provozieren. ${ }^{3}$

Für ausländische Vorbilder hinsichtlich Diagnose, Begutachtung oder Entschädigungspraxis gibt es in diesem Zusammenhang keine Belege oder starken Hinweise. Noch 1930 stellte der entsprechende Eintrag in der Enzyklopädie des Internationalen Arbeitsamtes fest, es gebe keine „special pathology resulting from pneumatic tools“ ([Loriga] 1930: 4). Vasomotorische Beschwerden („Totenfinger“), die in Italien, den USA und Großbritannien als Folge von vibrierenden Arbeitsmaschinen diskutiert wurden und 1929/30 vereinzelt ihren Weg in die deutsche Fachdebatte fanden ([Koelsch] 1929: 223-224; Hamilton 1930; Rainhorn 2014; Dembe 1996: 57-58), standen später im Schatten jener arthrotischen und nekrotischen Zustände der Gelenke, die Unfallchirurgen als „typisches“ Bild des Pressluftschadens gleichsam gegen die Gewerbemediziner durchsetzten. Anders als bei den Staublungenerkrankungen (Martin 2014: 230) konnte dem Ausschuss im Reichswirtschaftsrat daher kein ausgereifter Forschungsstand vorgelegt werden. Insofern scheint sich die rechtliche Setzung einem klinischen Einzelfall und der Erwartung verdankt zu haben, die starke Verbreitung von Pressluftgeräten werde künftig vermehrt zu Schäden führen, ${ }^{4}$ zumal der Status als Berufskrankheit die Arbeitgeber zu geeigneten Präventionsmaßnahmen verpflichtete.

In den ersten Jahren wurden weniger Ansprüche angemeldet als erwartet. Erst ab Mitte der 1930er Jahre wuchs die Zahl der erstmals entgoltenen Pressluftschäden stark an und bewegte sich bis in die 1960er Jahre hin- 
ein in der Bundesrepublik zwischen 500 und 1.500 pro Jahr (Stockhausen 1967: 181). Der Bergmannsheil-Chirurg Alois Laarmann, der 1944 die erste Monographie zum Preßluftschaden veröffentlichte, lobte rückblickend die Weitsicht der Gesetzgeber, eine vorerst nur potenziell relevante, sozialrechtlich konstruierte Berufserkrankung aufgenommen zu haben und damit „der ärztlichen Erkenntnis vorausgeeilt" zu sein (Laarmann 1944: 15). Genau das aber war das Problem für seine Vorgänger, die um 1930 die neue Berufskrankheit in ihre Begutachtungs- und Behandlungspraxis $\mathrm{zu}$ integrieren hatten.

Hier wie bei anderen Berufskrankheiten bildete die Verordnung nicht den Stand des medizinischen Wissens ab (Boyer 1995: 130; Hohmann 1997: 151-152). Im Hinblick auf die Pressluftschäden hatte die Zweite Verordnung aber geradezu eine Wissenslücke in die Welt gesetzt. Die Diskrepanz zwischen gesetzlichem Auftrag der Rentenbegutachtung und medizinischem Kenntnisstand setzte die Bergmannsheil-Chirurgen unter Zugzwang. Die folgende Passage aus einem Aufsatz des Oberarztes Paul Rostock zeigt, wie relativ geschmeidig eine jahrzehntelang eingespielte Beobachtungs- und Beurteilungsroutine auf die gesetzgeberische Zumutung reagieren konnte:

Nach Veröffentlichung der Verordnung trat an das Bergmannsheil die Notwendigkeit heran, sich darüber klar zu werden, um was für Erkrankungen es sich hierbei handelt. Denn es war zu erwarten, daß sehr bald die Aufforderung zur Beurteilung und Begutachtung derartiger Rentenansprüche an uns gerichtet werden würde. Eine Durchsicht des Schrifttums ergab nur sehr spärliche Resultate. [...]

Eine Anfrage beim Reichsarbeitsministerium direkt brachte uns in der Angelegenheit auch nicht wesentlich weiter. [...] Die einzige praktische Feststellung enthielt etwas Negatives, nämlich, daß Nervenerkrankungen nicht entschädigungspflichtig sind [...].

So blieb uns nichts weiter übrig, als auf dem Gebiete Erfahrungen zu sammeln, d.h. die Leute zu untersuchen und zu versuchen, aus der Summe der Beobachtungen Schlüsse zu ziehen. Um die Erfahrungen nicht $\mathrm{zu}$ verzetteln, wurden alle Leute, welche auf Grund der oben genannten Verordnung Rentenansprüche an die Sektion II der Knappschafts-Berufsgenossenschaft stellten, dem Bergmannsheil zur Untersuchung überwiesen. [...] Weiterhin wurden noch alle übrigen deutschen Berufsgenossenschaften aufgefordert, Leute mit derartigen Rentenansprüchen im Bergmannsheil untersuchen zu lassen oder die einschlägigen Akten zur Einsichtnahme zu übersenden. (Rostock 1931a: 284-285) 
Fachliteratur und amtliche Auskünfte stellten die Bergmannsheil-Ärzte nicht zufrieden. Hier dominierten die Kasuistiken Holtzmanns und anderer Gewerbeärzte (Beintker 1930; Seyring 1930), von denen sich Rostock in seinen Schriften ausdrücklich distanzierte, weil die dort geschilderten Krankheitsbilder für den Bergbau gänzlich untypisch seien (Rostock 1931a: 284, 1933: 93-95). Die zerrüttete Schulter von Holtzmanns Kesselschmied erschien jedoch prominent in den offiziellen Erläuterungen zur Zweiten Verordnung, Holtzmann verfasste auch den entsprechenden Abschnitt in den Ärztlichen Merkblättern über berufliche Erkrankungen und anderen Nachschlagewerken ([Koelsch] 1929: 223; Holtzmann 1930; Bauer \& Gerbis 1931: 235, Anm. 1). Eine weitere Orientierungsmarke für die Gutachter, die „dauernde Rechtsprechung“ der höchsten Spruchinstanz mit veröffentlichten Urteilen, kam erst um die Jahreswende 1929/30 in Gang. Als Schlüssel für die klinisch-unfallmedizinische Wissensgenerierung am Bergmannsheil erwies sich daher das bewährte Organisationsprinzip, die zu begutachtenden Personen zentral zusammenzuführen und durch einheitliche Beobachtung und Begutachtung, inklusive einer einheitlichen Aufzeichnungspraxis, vergleichbare Daten in relativ großer Menge zu produzieren.

Die begutachtende Klinik nahm den unausgesprochenen Forschungsauftrag der Gesetzgeber an und gewann - unter Zuhilfenahme des berufsgenossenschaftlichen Verwaltungsapparats und Berücksichtigung der Rechtsprechungspraxis - der gutachterlich noch diffusen Berufserkrankung ein vorläufig anwendungsfähiges Wissen ab. In dem zentralisierten Begutachtungsverfahren untersuchte Paul Rostock zahlreiche Menschen, die einen Zusammenhang zwischen einem körperlichen Leiden und ihrer Arbeit an pressluftgetriebenen Bohr- und Abbauhämmern herstellten, denn nur wenn Versicherte ihre Forderung aktiv an die Berufsgenossenschaft herantrugen, wurde daraus überhaupt ein Fall. Im April 1930 hatte er bereits 279 Antragsteller gesehen, und bis zum 23. März 1933 sollten insgesamt 833 Fälle zusammenkommen (Rostock 1930: 70, 1936b: 341). Wenn man nach Tabelle 1 zugrunde legt, dass landesweit bis Ende März 1933 etwa 1.045 Pressluftschäden gemeldet wurden, von denen Rostock 833 selbst untersucht hatte, waren dies gut 80 Prozent. Das entsprach im Großen und Ganzen dem Anteil, den Fälle der Sektion II am Gesamtaufkommen der Pressluftschäden ausmachten (Laarmann 1944: 18).

Den spärlichen Vorgaben des Gesetzestextes ließ sich in der engen Verknüpfung von Berufsgenossenschaft und Krankenhaus relativ schnell eine brauchbare Grundlage sowohl in gutachterlicher als auch in fachwissenschaftlicher Hinsicht abgewinnen (Boyer 1990: 52). Im Frühjahr 1930, ein gutes Jahr nach Erlass der Zweiten Verordnung, präsentierte Rostock erste Ergebnisse auf der Jahrestagung der Deutschen Gesellschaft für Chirurgie, 
Tab. 1 Zahl der gemeldeten und anerkannten Pressluftschäden

\begin{tabular}{llllll}
\hline & $\begin{array}{l}\text { Gemeldet } \\
\text { insge- } \\
\text { samt* }\end{array}$ & $\begin{array}{l}\text { Gemeldet } \\
\text { in Sekti- } \\
\text { on II* }\end{array}$ & $\begin{array}{l}\text { Anerkannt } \\
\text { insge- } \\
\text { samt* }\end{array}$ & $\begin{array}{l}\text { Anerkannt } \\
\text { in Sekti- } \\
\text { on II* }\end{array}$ & $\begin{array}{l}\text { Anerkennungen } \\
\text { in Sektion II** }\end{array}$ \\
\hline 1929 & 331 & 239 & 31 & 25 & 10,5 \\
1930 & 334 & 280 & 74 & 60 & 21,4 \\
1931 & 214 & 160 & 88 & 76 & 47,5 \\
1932 & 135 & 101 & 76 & 60 & 59,4 \\
1933 & 119 & 92 & 69 & 55 & 59,8 \\
1934 & 147 & 119 & 84 & 75 & 63,0 \\
1935 & 166 & 138 & 77 & 69 & 50,0 \\
1936 & 270 & 234 & 110 & 94 & 40,2 \\
1937 & 409 & 329 & 203 & 178 & 54,1 \\
1938 & - & 697 & - & 356 & 51,1 \\
1939 & - & 894 & - & 502 & 56,2 \\
1940 & - & 1.014 & - & 446 & 43,9 \\
1941 & - & 1.553 & - & 599 & 38,6 \\
\hline
\end{tabular}

Quellen: Bürkle de la Camp (1939a: 162); Laarmann (1944: 18, Tab. 2)

*in absoluten Zahlen; **in Prozent

vor der Medizinischen Gesellschaft Bochum und der Rheinisch-Westfälischen Röntgengesellschaft (Rostock 1930, 1931a: 290, Literaturhinweise 5 und 6). Im Oktober 1930 waren die ersten Fachartikel zum Thema Pressluftschäden abgeschlossen (Wette 1931; Rostock 1931a). Knapp zwei Jahre später erschienen die Kenntnisse über das zuvor unbekannte Berufsleiden so weit abgesichert, dass Rostock eine erste, wenngleich mit Disclaimern versehene Zusammenschau für das von Georg Magnus mit herausgegebene Handbuch der gesamten Unfallheilkunde abliefern konnte. Dieser Beitrag dürfte das gutachterlich-sozialrechtliche Wissen in Bezug auf Pressluftschäden weiter konsolidiert haben, währen die wissenschaftlich-ätiologische Diskussion erst am Anfang stand. Rostock betonte, die Pathogenese der beobachteten Schädigungen stelle immer noch „ausgesprochenes medizinisches Neuland“ dar (Rostock 1933: 111). Um die Wirkung von Maschinenarbeit auf den Körper zu erforschen, seien neben weiterer klinischer Beobachtung vor allem experimentelle Untersuchungen nötig.

\section{„Preßluftellebogen“ made in Bochum, 1929-1934}

Durch die Zweite Verordnung, so befand Paul Rostock später, sei „das Studium der Gelenkschäden sehr erheblich gefördert worden“ (Rostock 1936a: 
303). Ausgangspunkt für diesen allgemeinen Wissenszuwachs war aber zunächst die Suche nach klinischen und vor allem röntgenologischen Beurteilungskriterien für die Begutachtung der Berufskrankheit. Dazu mussten sich die Chirurgen ein Bild von Typik, Bandbreite und Verlauf zu entschädigender Leiden machen.

Ausgehend von der Häufigkeit der Schädigungen sah Rostock in erster Linie die Ellenbogengelenke als betroffen an, mit deutlichem Abstand auch die Handgelenke. Aufgrund des röntgenologischen Erscheinungsbildes der Ellenbogen ist wechselweise von Arthritis oder Arthrosis deformans die Rede. Um die damit bezeichneten Abnutzungs- und Kompensationsprozesse der Verkalkung und Verknöcherung von alters- oder konstitutionsbedingtem Um- und Abbau abzugrenzen, musste überprüft werden, ob Gelenke in verschiedenen Regionen des Körpers krankhaft verändert waren oder nur an Stellen mechanischer Einwirkung. Differentialdiagnostisch stellte sich zudem die Aufgabe, „für einzelne Gelenke besonders charakteristische Arthritisformen (sei es in bezug auf Lokalisation im Gelenk oder röntgenologische Form der Veränderung oder klinische Erscheinungen usw.) zu finden, von denen man annehmen kann, daß sie Folge der Preßluftwerkzeugarbeit sind“ (Rostock 1933: 93).

Die im Röntgenbild beobachteten anatomischen Veränderungen am Ellenbogen unterteilte Rostock zunächst in zwei morphologische Grundformen, und zwar die Bildung von Knochenzacken im Bereich des Gelenks, vor allem an der Beugeseite des Oberarmknochens, sowie ein verbreitertes und überhängendes Radiusköpfchen. Später unterschied er ätiologisch zwei Schädigungsvarianten. Zum einen Prozesse der Knochenwucherung und Verknöcherung an Muskelansatzpunkten und Gelenkkapsel, die dadurch entstanden, dass die Erschütterung des Arbeitsgeräts muskulär abgefedert wurde und der Körper die Degeneration der Bindegewebe kompensierte, wobei er Zacken an der Beugeseite des Oberarms weiterhin für „fast absolut pathognomisch“ erklärte (Rostock 1934b: 632). Als zweites identifizierte er Nekrosen, die durch Aufeinanderdrücken der Gelenkflächen entstanden und im Handgelenk zum Mondbeintod führten, im Ellenbogen Gelenkkörper aussprengten beziehungsweise die Gelenkflächen umformten (Rostock 1936b: 341).

Im Hinblick auf den Verlauf der einzelnen Schädigungsformen versuchte Rostock ein „Gesamtbild“ des Phänomens aus unterschiedlichen Einzelfällen zusammenzusetzen. Denn da Arbeiter mit entsprechenden Gelenkschäden den Arbeitsplatz wechseln mussten, werde es „wohl nie möglich sein, über Jahre hinaus an einem Mann die Entwicklung der Veränderungen zu verfolgen“ (Rostock 1931a: 286, Hervorhebung im Original). Um sich ein „Bild davon [zu] machen, wie wohl das Fortschreiten von leichten Formen zu den schwersten Veränderungen vor sich gegangen ist“, mussten „eine 
größere Zahl von Beobachtungen“ übereinandergelegt werden (Rostock 1933: 96-97). Dies war ganz praktisch zu verstehen. In seiner Veröffentlichung zeigte Rostock Röntgenaufnahmen unterschiedlicher Ellenbogengelenke, die aufeinanderfolgende Stadien des angenommenen Krankheitsverlaufs repräsentierten. 1934 konnte Rostock erstmals einen Bergmann untersuchen, der schon längere Zeit an Pressluftschäden litt und dauernd weitergearbeitet hatte. In einer Schemazeichnung führte Rostock nun einen älteren und einen aktuellen Röntgenbefund zusammen, um den Verlauf der Knochenwucherung in einem einzigen Bild darzustellen (Rostock 1934a: 74).

Seit 1910 bekannt war dagegen der Verlauf der zweithäufigsten anerkannten Schädigung, der Lunatumnekrose (Mondbeintod), dem Absterben eines Handwurzelknochens. Da jedoch die Pathogenese umstritten blieb, ging es hier vor allem darum, mit den bewährten Faustregeln der Unfallversicherung jene Umstände abzugrenzen, unter denen dieses in der Allgemeinbevölkerung vorkommende Leiden als Berufskrankheit mit Pressluftarbeit in Zusammenhang gebracht werden könne (Rostock 1933: 102-103). Da das Mondbeinleiden bei Bergmännern überproportional häufig auftrete, hielten die Bergmannsheil-Chirurgen einen Zeitraum von zwei Jahren dauerhafter Arbeit an Pressluftwerkzeugen für eine robuste Voraussetzung, um eine Berufserkrankung anzuerkennen (Wette 1931: 313-314; Rostock 1931b: 346).

Auch bei den weitaus häufigeren Schäden am Ellenbogen wurde „dem Arzt besonders von den technischen Beratern der Berufsgenossenschaft, aber auch von den Juristen der Spruchbehörden die Frage gestellt, wie lange ein Arbeiter Preßluftwerkzeuge bedient haben muß, wenn er durch diese Arbeit Gelenkschäden sich erworben haben will“" (Rostock 1931b: 337). Im Sommer 1931 schien mit bis dato 520 untersuchten Fällen genug Material zusammengekommen zu sein, um diese Frage untersuchen zu können. Der medizinische Befund wurde mit den Arbeitszeiten an entsprechenden Werkzeugen abgeglichen, die Beamte der Berufsgenossenschaft zusammenstellten, indem sie Arbeiter befragten und in den Arbeitsbüchern der Zechen recherchierten. Der Abgleich kam zu dem Ergebnis, dass es keine statistische Korrelation zwischen Art und Dauer der Pressluftarbeit und dem Ausmaß der Schädigungen gebe. Nicht wenige Bergleute hatten Jahrzehnte Pressluftarbeit ohne Beeinträchtigung überstanden, während andere schon nach relativ kurzer Zeit unter Beschwerden litten, die sich röntgenologisch darstellen ließen. Als Arbeitsformel empfahl Rostock daher, bei der Entschädigung eine Bedienungszeit von mindestens zwei Jahren zugrunde zu legen (Rostock 1931b: 346).

Welche biomechanischen Prozesse genau in der Schädigung durch Pressluftwerkzeuge wirksam wurden, blieb umstrittener Gegenstand theo- 
retischer Überlegungen. Dennoch ließ sich dem Problem ein provisorisches Begutachtungswissen abgewinnen, das den Umgang mit entschädigungspflichtigen Beschwerden ermöglichte. Denn es stellte pragmatische Antworten auf die zentrale Frage nach dem Kausalzusammenhang bereit, deren Grundmuster sich in der unfallrechtlichen Beurteilung von ganz unterschiedlichen Leiden wie Gehirnerschütterungen oder Leistenbrüchen über Jahrzehnte eingebürgert hatten (Barta 1983).

Das betraf vor allem das als "typisch" herausgearbeitete Bild, die isolierten Schäden an den Ellenbogengelenken. Ihre Berentung verlief innerhalb des berufsgenossenschaftlichen Entschädigungsverfahrens offenbar vergleichsweise konfliktfrei (Thomann 1993: 326, 342), so dass sich Alois Laarmann später (1944: 16) von dieser „mehr oder weniger mechanischen Anwendung des Begriffes vom Preßluftschaden" distanzierte und damit indirekt die Vertrauensärzte kritisierte: „Preßluftarbeit einerseits oder der vermeintlich typische Befund andererseits, bestenfalls noch das Zusammentreffen dieser beiden Anhaltspunkte genügte vielfach, um die Diagnose zu stellen bzw. einen Zusammenhang ohne weiteres zu bejahen".

Neben der Benennung des „Typischen“ gab es jedoch frühzeitig eine Weitung ins Allgemeinere und damit ins gutachterlich Uneindeutige. War Rostock nach eigener Aussage in den ersten Monaten seiner Recherche zunächst auf der Suche nach dem charakteristischen „Preßluftellebogen“ des Bergmanns gewesen, kam er davon bald ab. Denn in der Literatur und in eigener Anschauung traf er auf identische Schadensbilder, die bei vergleichbarer mechanischer Belastung, jedoch ohne den Gebrauch von Pressluftwerkzeugen zustande gekommen waren. Bereits in seinen ersten Veröffentlichungen (Rostock 1931a: 284) übernahm er daher das unspezifische Konzept der "funktionellen Überinanspruchnahme“, die er später (Rostock 1933: 102) auch als „Missverhältnis der Belastung und Widerstandsfähigkeit des Gelenks" beschrieb. Der "typische Preßluftellebogen“ bewährte sich als diagnostische Formel bei der Begutachtung relativ klarer Fälle. Zugleich wurde die Berufskrankheit Pressluftschaden zu einem generalisierbaren „Musterbeispiel“, an dem "die pathologischen Veränderungen und die Reaktionsweise des Körpers auf die Erschütterungen am besten zu studieren" seien (Rostock 1934b: 634), unabhängig von sozialrechtlichen Fragestellungen. 


\section{Gutachten in der Rechtsprechung, 1929-1934}

Bei aller gebotenen Skepsis gegenüber der Wissenstechnik Statistik deuten die in Tabelle 1 aufgeführten Zahlen auf eine rasche Verfestigung des Begutachtungswissens in den ersten Jahren nach Erlass der Zweiten Verordnung hin. Von den erstmals bei der Sektion II gemeldeten Pressluftschäden wurden 1929 gerade einmal 10,5 Prozent als entschädigungspflichtige Berufskrankheit anerkannt. Diese Quote verdoppelte sich im folgenden Jahr und lag zwischen 1931 und 1939 im Schnitt bei 53,5 Prozent. Gut die Hälfte aller internen Verfahren endeten in diesem Zeitraum also mit der Bewilligung einer Erwerbsminderungsrente von meist 20 bis 30 Prozent.

Reibungen entstanden in Fällen, die aus dem Bild des als „typisch“ und daher entschädigungspflichtig Festgestellten herausfielen. Schon der Kommentar zur Zweiten Verordnung hatte deutlich gemacht, dass die Bandbreite der auftretenden Gesundheitsschädigungen im Zusammenhang mit Pressluftarbeiten deutlich größer sei als der Kreis zu entschädigender Leiden ([Koelsch] 1929). Viele Krankheitsbilder wurden in den Gerichtsverfahren der unteren Spruchinstanzen bis zum Knappschaftsoberversicherungsamt als Folge von Pressluftschäden ausgeschlossen, Senkfuß ebenso wie Sehnenscheidenentzündung oder Morbus Parkinson (Leitfaden 1934: 92, 96-97, 99). Andere Grenzfälle, darunter auch die häufig fragliche Abgrenzung von degenerativen Gelenkerkrankungen kamen vor das Reichsversicherungsamt. Wie bei der Silikose (Martin 2002: 108) ist anzunehmen, dass auch hier über die höchstinstanzlichen Rekursverfahren „Kriterien des entschädigungspflichtigen Krankheitsbildes entwickelt" wurden. Als die Landesgewerbeärzte 1930 über eine weitere Ausdehnung der Berufskrankheitenliste berieten, hielten sie es noch für möglich, die bisher nicht erfassten Erkrankungen der Sehnen, Nerven und Gefäße „im Wege der Rechtsprechung [...] nach dem jetzigen Wortlaut unter die V[erordnung] zu bringen". 5 Auch den Gutachtern war klar, dass der Gesetzestext wesentlich über richtungsweisende Einzelfallentscheidungen auslegt wurde.

In Laarmanns Monographie von 1944 und zwei berufsgenossenschaftlichen Leitfäden zur Begutachtung aus den 1930er Jahren sind 36 Entscheide des Reichsversicherungsamts von März 1930 bis Oktober 1934 aufgeführt, die als relevant für die Entschädigungspraxis in Pressluftfällen angesehen wurden (Leitfaden 1934: 88-101; Leitfaden 1937: 169-189; Laarmann 1944: 106-123). Davon stammten 24 Fälle aus der Sektion II. Die Tendenz dieses Samples ist eindeutig: Von den 36 Entscheiden schlossen 33 eine Entschädigung bestimmter Leiden grundsätzlich oder fallweise aus, überwiegend Gelenkerkrankungen und Muskelschwund, aber auch die Dupuytrensche Kontraktur, Mondbein- und Meniskusleiden sowie Chondromatose. Damit waren Entscheide gegen die Versicherten allerdings überrepräsentiert, 
denn einer Auswertung von rund 1.600 Verfahren zufolge erkannte der Senat für Berufskrankheiten des Reichsversicherungsamts bis einschließlich 1933 etwa 20 Prozent aller Pressluftschädigungen an (Sniegs 1993: 211-212).

Interessanter als die tendenziöse Auswahl ist hier jedoch die Frage, wie sich das im Bergmannsheil unter monopolartigen Bedingungen (Thomann 1993: 231) hervorgebrachte Begutachtungswissen in den Entscheidungen dieser höchsten Spruchinstanz bewährte. Die Gutachten der Bergmannsheil-Chirurgen liegen den überlieferten Rekursunterlagen nur ausnahmsweise bei, da sie meist in einem früheren Stadium des Verfahrens in Auftrag geben worden waren und zu den vorgängigen Akten gehörten. Trotz ihrer herausragenden empirischen Expertise bei Pressluftschäden wurden Bergmannsheil-Chirurgen selten mit Obergutachten beauftragt. Qua institutioneller Stellung galten ihre Beurteilungen offenbar nicht als ausreichend überparteilich im Sinne des auf Interessenausgleich zielenden Auftrags der Sozialversicherung. Zwar attestierte das Reichsversicherungsamt ihnen 1934 „eine besondere Sachkenntnis auf diesem Gebiete“ und in der Tat folgen zahlreiche Urteilsbegründungen den Gutachten. ${ }^{6}$ Unkritisch gingen die Entscheider aber wohl nicht vor. Michael Bauer, Ministerialrat im Reichsarbeitsministerium und ärztlicher Beisitzer im Senat für Berufskrankheiten, bezeichnete 1932 in einer internen Notiz die Bochumer Begutachtungspraxis als „zumindest merkwürdig“:

Es wird fast immer festgestellt, dass der betreffende Antragsteller an einer Arthritis leidet und dass diese Arthritis nicht die typischen Zeichen einer Schädigung durch Preßluftwerkzeuge aufweise. Ich habe aber noch nicht gefunden, welche Schädigungen das Krankenhaus als „typisch“ bezeichnet. Mir wenigstens scheint es angezeigt zu sein, diesen Begutachtungen in der nächsten Zeit ein besonderes Augenmerk zuzuwenden. ${ }^{7}$

Das Changieren des Pressluftellenbogens zwischen „Typischem“ und nicht Typisierbarem, ihre Doppelfunktion als Gutachter und Wissenschaftler konnte die Bergmannsheil-Chirurgen auch in konkurrierenden Gutachten einholen. In einem Fall von freien Körpern im Bereich des Ellenbogengelenks argumentierte der vom Kläger vorgeschlagene Gutachter, Chefarzt am Evangelischen Krankenhaus Sterkrade, geschickt für eine Entschädigungspflicht, indem er einerseits feststellte, dass es bisher nicht gelungen sei, ein „charakteristisches Bild für Gelenkstörungen durch Preßluftwerkzeuge zu präzisieren“, sodass freie Körper nicht einfach als „untypisch“ ausgeschlossen werden könnten. Andererseits führte er an, dass es bei dem Kläger neben den Gelenkkörpern auch Hinweise auf die von Rostock 
als „typisch“ beschriebenen Zeichen wie ein verbreitertes Radiusköpfchen und eine arthritische Zacke an der Beugeseite des Oberarmknochens gebe. ${ }^{8}$ Dem schloss sich der Senat an.

Gegenwind hatten Begutachtungen aus dem Hause Bergmannsheil insbesondere von Klägern zu erwarten, die sich von gewerkschaftlichen Arbeitersekretariaten beraten und vor Gericht vertreten ließen (Shin 2007: 89-102, 178-182). Auf Wunsch von klagenden Versicherten forderte das Reichsversicherungsamt nicht selten Obergutachten bei prominenten Gewerbe- und Versicherungsärzten wie Ludwig Teleky, Erich Beintker oder Hans Liniger an. ${ }^{9}$ Beauftragte der Senat für Berufskrankheiten aus eigenem Antrieb ein Obergutachten, dann wandte er sich eher an Universitätsmediziner, bei Pressluftschäden im Ruhrgebiet an die chirurgische Universitätsklinik Münster. ${ }^{10}$

Wie gerade die umstrittenen Fälle zeigen, die am Ende gegen die Kläger entschieden wurden, ging es weniger darum, den Versicherten durch eine lange Verfahrensdauer Leistungen vorzuenthalten, als vielmehr den widerstreitenden medizinischen Auffassungen im Verfahren Raum zu geben. ${ }^{11}$ In einem Fall schloss sich der ärztliche Beisitzer Ernst Wilhelm Baader, Leiter der klinischen Abteilung für Gewerbekrankheiten am Berliner AugusteViktoria-Krankenhaus, zunächst der Arthritisdiagnose des Bergmannsheil an und plädierte dafür, das Rechtsmittel des Klägers ohne mündliche Verhandlung zurückzuweisen. Drei Tage später revidierte er seine Empfehlung, da er zwar selbst nach wie vor keinen Zusammenhang zur Berufsarbeit sehe, unterdessen aber den Vortrag eines prominenten Unfallchirurgen gehört habe, der die traumatische Auslösung von Arthritis für möglich halte. Offenbar kam es nicht allein auf die Stichhaltigkeit der in Gutachten präsentierten Argumente an, sondern es galt auch, bei der Entscheidungsfindung allen legitimen Einwänden ein Forum zu bieten. ${ }^{12}$

Überdies waren medizinische Gutachten lediglich ein Mittel der freien Beweiswürdigung, das von übergeordneten Gerechtigkeitsannahmen ausmanövriert werden konnte. 1930 verurteilte das Reichsversicherungsamt die Berufsgenossenschaft zur Entschädigung eines Bergmanns, dem nach einer Kniegelenksvereiterung der Unterschenkel hatte amputiert werden müssen. Der Versicherungsträger hatte nach Erlass der Zweiten Verordnung zunächst einige Fälle von Pressluftschaden am Knie anerkannt, weil Bergleute die Presslufthämmer teilweise oberhalb des Knies abstützten (Kelzenberg 1941: 34). In diesem Fall betonte das Bergmannsheil-Gutachten jedoch, das Leiden sei von einem Schleimbeutel unterhalb des Knies ausgegangen, der anatomisch nicht zum Kniegelenk gehöre, weshalb keine Entschädigungspflicht bestehe. Die Mitglieder des Senats für Berufskrankheiten erörterten die in verschiedenen Gutachten aufgestellten Kausalzusammenhänge der Infektion - Schleimbeutelentzündung, Erguss in Knie- 
gelenk oder Hautabschürfung -, bis der Senatspräsident ein Machtwort sprach, das die mehrdeutigen medizinischen Indizien in den Hintergrund drängte: „Dem Mann muß geholfen werden. Mit subtilen Erwägungen [...] kommt man m.E. an die Sache nicht gut heran". ${ }^{13}$

Der Fall fand als wichtige Referenz Eingang in die Entscheidungen und Mitteilungen des Reichsversicherungsamts (1930: 27, 1930: 505-506) und andere Sammlungen. Erledigt war die Frage nach dem Zusammenhang von Pressluftarbeit und Erkrankungen des Knies damit nicht, zumal die Position der Bergmannsheil-Chirurgen, nur Schäden an den Arbeitsarmen kämen für Rentenzahlungen in Betracht, ein gewichtiges Gegenargument darstellte. Die weitere Diskussion vor allem über Meniskusrisse zeigt jedoch, dass Gutachter, die den Gesetzestext zugunsten der Berufsgenossenschaft in der Regel eng auslegten, gewissermaßen beiläufig neue Entschädigungstatbestände schaffen konnten.

Die im Bergbau häufig vorkommenden Schädigungen der Knorpelzwischenscheiben im Knie waren lange als beruflicher Unfall anerkannt worden, bis in den 1920er Jahren histologische Reihenuntersuchungen langwierige Degenerationsprozesse im Knieknorpel beschrieben. Am Bergmannsheil beschäftigte sich der Assistenz- und spätere Oberarzt Remmer Andreesen seit Anfang der 1930er Jahre klinisch und pathologischanatomisch mit den Meniskusleiden. Aus seiner Begutachtungsperspektive erschienen gerade leichtere Unfälle oder alltägliche Bewegungen (wie das Aufstehen) bei bereits degenerierten Menisken nur mehr als auslösender "Gelegenheitsanlaß" und nicht als Ursache von Verletzungen.

Darüber entwickelte sich ein Gutachterstreit, in dem sich medizinische Auffassungen und die Ökonomien verschiedener Versicherungsträger überlagerten. Denn der Opponent der Bergmannsheil-Chirurgen war Fritz Linde, Chefarzt der chirurgischen Abteilung am Knappschaftskrankenhaus in Gelsenkirchen. Die Knappschaft als Trägerin der Kranken-, Alters- und Invalidenversicherung der Bergleute musste zahlen, wenn die Berufsgenossenschaft eine Verletzung oder Erkrankung nicht als Unfallfolge oder Berufskrankheit anerkannte. Linde wollte die Erschütterungswirkung von Pressluftgeräten am Knie als „chronisches Trauma“ verstanden wissen ein Hybrid aus dem einmaligen Ereignis des Unfalls und der dauerhaften Belastung einer Berufskrankheit. „Die Meinungsverschiedenheiten unter den Ärzten in der Frage der Entstehung des Bandscheibenrisses“, so Linde, „haben zu nichtendenwollenden Rechtsstreitigkeiten zwischen der Ruhrknappschaft und der Knappschaft-Berufsgenossenschaft geführt" (Linde 1935: 942).

Die Argumentation des Bergmannsheil in dieser Sache setzte sich vermutlich vor dem Reichsversicherungsamt überwiegend durch (Andreesen 1937a, 1937b; Leitfaden 1937: 180; Anonymus 1938). Allerdings führten die 
abschlägigen Begutachtungen von Arbeitsunfällen und Berufskrankheiten wiederum zu Beobachtungen, die nach Konsequenzen verlangten. Schon 1932 gelangte man am Bergmannsheil zu der Auffassung, das abgenutzte „Bergarbeiterknie“ müsse als eigenständige Berufskrankheit verstanden werden, da es sich um eine unter Bergleuten allgegenwärtige erworbene Konstitution handele (Andreesen 1934a: 59). Fachöffentlich forderte Andreesen 1933 auf der Tagung der Deutschen Gesellschaft für Chirurgie, „im Interesse der schwerarbeitenden Bergleute die betreffenden Behörden und Regierungsstellen darauf hinzuweisen“, dass die Degeneration der Menisken für bestimmte Berufsgruppen dem gesetzlichen Katalog der entschädigungspflichtigen Berufskrankheiten hinzuzufügen sei (Andreesen 1934a: 72; vgl. auch Andreesen 1934b: 1.235, 1937a, 1937b; Laarmann 1936). Die chirurgischen Gutachten aus dem Bergmannsheil ersparten der Berufsgenossenschaft Rentenzahlungen, weil sie Meniskusrisse nicht als Folge von Pressluftarbeit anerkannten. Gleichsam als Nebenprodukt dieser Argumentationsarbeit baute sich mit den Meniskusschäden als potenzielle Berufskrankheit aber ein sehr viel größerer finanzieller Posten auf, wenngleich er erst nach dem Zweiten Weltkrieg in beiden deutschen Staaten in die Entschädigungspflicht eingebaut werden sollte.

\section{Verschiebungen 1934-1944}

Etwa fünf Jahre nach Erlass der Zweiten Verordnung waren die Pressluftschäden sozialrechtlich weitgehend vermessen. Danach zirkulierten kaum noch neue Entscheide des Reichsversicherungsamtes in dieser Sache, und 1934 veröffentlichte die Knappschafts-Berufsgenossenschaft den bereits erwähnten Leitfaden zur Zweiten Verordnung, in dem für jede Nummer der Berufskrankheitenliste wichtige Entscheidungen der Spruchbehörden bzw. wissenschaftliche Veröffentlichungen aus Medizin und Bergbautechnik zusammengestellt waren, teils versehen mit kurzen Auszügen, Kommentaren und Merksätzen. Zusammengetragen aus unterschiedlichen Quellen, zu einem nicht geringen Teil aus den eigenen Unfallakten, bildete der Leitfaden eine Art Handapparat der Entscheidungsfindung mit einem kasuistischen Fundus. Der hier präsentierte Wissensstand war nicht nur für den internen Gebrauch bestimmt, sondern adressierte laut Titel auch „Krankenkassen, Kreis-, Gewerbe- und andere Ärzte, Gewerbeaufsichtsbeamte, Versicherungs- und Oberversicherungsämter, Landesversicherungsanstalten, Krankenhäuser usw..

Gegenüber dieser Konsolidierung des gutachterlichen Wissens ergab sich auf der personellen Ebene ein Einschnitt. Im November 1933 wech- 
selte Georg Magnus nach Berlin und übernahm die Leitung der II. Chirurgischen Klinik in der Ziegelstraße. Paul Rostock und weitere Mitarbeiter folgten ihm, die in den folgenden Jahren aus der Klinik eine Kaderschmiede der NS-Medizin machten (Lammel 1994: 583; Schmiedebach \& Schwoch 2001a: 36-41). Obwohl der Ortswechsel Rostock von seinem „Material“ in der Bergbauregion trennte, deckte er das Thema weiterhin auf Fortbildungskursen zu Berufskrankheiten $a b,{ }^{14}$ publizierte und referierte auf Kongressen zur erweiterten Frage der „chronische[n] Erschütterungen“ und „abnorm starke[n] Arbeitsbeanspruchung“ (Rostock 1934b; 1935b; 1936a; 1936b). Gelegentlich erstattete er auch noch Gutachten zu Pressluftschäden für die Sektion II (Leitfaden 1937: 180, 185).

Unterdessen ging am Bergmannsheil die institutionalisierte Expertise auf Magnus' Nachfolger Heinrich Bürkle de la Camp über, der sich bis dato nicht mit Pressluftschäden befasst hatte. Zusammen mit Remmer Andreesen und Alois Laarmann setzte er den Begutachtungsbetrieb fort und versorgte die Fachöffentlichkeit und praktische Ärzte mit Fortbildungskursen und Überblicksartikeln in Zeitschriften und Handbüchern (Bürkle de la Camp 1937, 1938, 1939b; Andreesen 1942a; 1942b). Neue Akzente setzten unter anderem die bereits von Rostock eingeforderten experimentellen Forschungen, um die Ätiologie der Pressluftschäden weiter aufzuklären. Röntgenaufnahmen von „arbeitenden“ Ellenbogen- und Handgelenken wurden mit ruhenden verglichen, um die Mechanik der Abfederung von Pressluftstößen zu beobachten (Laarmann 1944: 22-23, 52-53). Für die Lunatumnekrose wurde bei amputierten Händen oder Armen Quecksilber in die Blutgefäße injiziert und die Durchblutung in verschiedenen Stellungen beobachtet (Köstler 1935; Laarmann 1944: 55-57).

Zudem rückte die Frage der "Konstitution“ in den Fokus, die Rostock angesichts der fehlenden Korrelation zwischen Arbeitsbelastung und Schädigung bereits aufgeworfen hatte. Zwar fand man am Bergmannsheil in der zweiten Hälfte der 1930er Jahre keine Konstitutionstypen, die sich für Pressluftarbeit als (un)geeignet erwiesen, dennoch stand die unterschiedlich ausgeprägte „Krankheitsbereitschaft des Körpers“ stark im Vordergrund (Andreesen 1942a: 123, 126). Die Ärzte sprachen von „Bindegewebs-“ und "Gelenkschwächlingen“ (Laarmann 1940: 65), da Schäden im Bild des „Pressluftellenbogens" mittlerweile nicht nur in anderen Berufen, sondern auch bei Hausfrauen und Schülern beobachtet worden waren, die keine mechanische Schwerstarbeit leisteten (Laarmann 1944: 34).

Nachdem im Bergbau so genannte „Anlegeuntersuchungen“ vorrangig mit Blick auf die Silikose bei der Einstellung verpflichtend geworden waren, nahmen auch die Chirurgen probeweise solche prophylaktischen Screenings an 200 Personen vor, die nicht oder nur kurzzeitig mit Pressluftwerkzeugen gearbeitet hatten. Schon in der Gruppe der bis zu 24-Jähri- 
gen stießen sie auf Befunde, die sie als angeborene Gewebeminderwertigkeit interpretierten, und empfahlen sowohl eine restriktivere Begutachtung als auch eine verbesserte Personalauslese durch Berufsberatung: „Bindegewebsschwächlingen und solchen Leuten, die man als besonders empfindlich für den Erschütterungsreiz erkannt hat", solle die Arbeit an Pressluftwerkzeugen untersagt und sie anderweitig eingesetzt werden (Bürkle de la Camp 1937: 1351, 1939a: 163).

Obwohl mit all diesen Untersuchungen „der exakte Nachweis über schädigende Wirkung des Preßluftwerkzeuges auf das menschliche Gewebe“ nicht zu erbringen war (Andreesen 1942a: 121), untergruben sie doch teilweise die Prämissen der bisherigen Begutachtungspraxis. Durch den Wissenszuwachs, so Laarmann, müsse die „Zusammenhangsbegutachtung des Preßluftschadens“ aktuell „als wesentlich schwieriger bezeichnet werden, als das in früheren Jahren zunächst angenommen wurde" (Laarmann 1944: 95). In manchen Fällen schwer entstellter Gelenke sei der Pressluftschaden bisher nur eine Verlegenheitsdiagnose gewesen (Bürkle de la Camp 1939a: 166; Laarmann 1940: 65). Zudem förderten katamnestische Studien eine Reihe an „Fehlurteilen“ in früheren Gutachten zutage (Kelzenberg 1941: 32-35; Laarmann 1944: 78-80; Andreesen 1942a: 127, Abb. 7). Auf der XIII. Tagung der Deutschen Gesellschaft für Unfallheilkunde forderte Bürkle de la Camp daher, die Entschädigungspraxis bei Pressluftschäden müsse sowohl erweitert als auch eingeengt werden. Verschärfend müsse im Hinblick auf die Differenzialdiagnostik von Allgemeinerkrankungen vorgegangen werden, um insbesondere chronische Infektionen, etwa im Gebiss oder in den Harnwegen, auszuschließen, was dazu führen werde, Pressluft nicht mehr oder nur noch als Teilursache des Leidens anzuerkennen. Dafür sei eine stationäre Untersuchung mit Blutbild und Blutsenkung nötig (Bürkle de la Camp 1939a: 162-163). Eine Ausweitung forderte Bürkle de la Camp im Hinblick auf die Geräte selbst, da bislang nur Berufserkrankungen durch transportable Pressluftwerkzeuge als entschädigungspflichtig galten. Weiter plädierte er dafür, die Exponierungsdauer als Voraussetzung für eine Anerkennung herabzusetzen (Bürkle de la Camp 1939a: 164, 166). Und schließlich hielt er die „im Schrifttum mehrfach empfohlene Erweiterung des Gesetzes auf Erkrankungen der Nerven und Blutgefäße" in einer engen Auslegung für möglich (Bürkle de la Camp 1939a: 168), zumal seine eigene Abteilung in Einzelfällen solche Leiden als Berufskrankheit anerkannte (Bürkle de la Camp 1937: 1350, 1939a: 167; Andreesen 1942a: 122).

Lange hatte das Bergmannheil diese alte Forderung der Gewerbeärzte in Gutachten und Publikationen bekämpft. Es hatte Aufsehen erregt, als das Reichsversicherungsamt im April 1931 in einem Fall von Muskelschwund durch Nervenlähmung den angefochtenen Bescheid der Berufsgenossenschaft mit der Erläuterung aufgehoben hatte, unter den Begriff Muskel im 
Sinne des Gesetzes falle „nicht nur das anatomisch als solche anzusehende Körpergewebe, sondern es sind auch die den Muskel versorgenden peripheren Nerven einzubeziehen, beide also für die Beurteilung der gewerblichen Schädigung als Einheit zu betrachten“. ${ }^{15}$ Obwohl die Angelegenheit an die Berufsgenossenschaft zurücküberwiesen wurde, hatte dieser vielfach nachgedruckte und zitierte Entscheid Auswirkungen. Er veränderte die Geschäftsanweisung für Knappschaftsärzte ${ }^{16}$, und Gutachter bezogen sich auf ihn, um vereinzelt den Auslegungsspielraum des Gesetzestextes auszuweiten (Hennig \& Gallinek 1933).

Gegen den Entscheid hatte Paul Rostock zwar auf dem scharf abgegrenzten Wortlaut der Verordnung beharrt, was eine grundsätzliche Entscheidung des Reichsversicherungsamtes in Bezug auf Muskelschwund 1933 auch bestätigte. ${ }^{17}$ Diese bestätigende Entscheidung revidierte jedoch nicht das vorherige, ausweitende Urteil - beide standen nebeneinander, und auch Rostock erkannte die sozialrechtliche Relevanz des Entscheids von 1931 an, indem er ihn in seinem Handbuchbeitrag und später in einem Leitfaden zur Unfallbegutachtung referierte (Rostock 1933: 107-108, 1935a: 97). Umgekehrt gab es die Einzelfallprüfung her, ein Nervenleiden ausnahmsweise unter Berufung auf Rostock für anerkennungswürdig zu erklären, wenn es als Folge der „typischen“ beruflichen Deformierung des Ellenbogengelenks im fortgeschrittenen Stadium auftrat (Weber 1934: 79).

Es waren jedoch vor allem Gewerbemediziner, die darauf drangen, auch andere Gewebsarten in die Entschädigungspflicht einzubeziehen. Pressluftgeschädigte Nerven und Gefäße erhielten den Status einer faktischen „Berufserkrankung“ - wenn auch nicht eines sozialrechtlichen Tatbestands - in gewerbehygienischen Handbüchern und der Kommentarliteratur, so in einem Band der Deutschen Arbeitsfront zur Dritten Berufskrankheitsverordnung von 1936 (Hebestreit \& Bartsch 1937). Ab Mitte der 1930er Jahre scheinen Gutachter häufiger für eine Ausweitungen auf Nerven und Gefäße plädiert zu haben, wenn die Handhabung von Pressluftgeräten die plausibelste Erklärung für eine Symptomatik bot (Junghanns 1936; Schramm 1938: 255-256). Selbst Georg Magnus, der zu diesem Zeitpunkt allerdings seit fünf Jahren nicht mehr im Dienst des Bergmannsheil stand, sprach sich 1938 auf dem VIII. Internationalen Kongress für Unfallmedizin und Berufskrankheiten in Frankfurt am Main für eine liberale Interpretation in wenigen begründeten Fällen aus, denn es sei „sehr möglich, daß man mit einer derartigen Auffassung den Sinn des Gesetzes besser trifft, als wenn man nur dem Wortlaut genau folgt" (Magnus 1939: 631).

Die Dritte Verordnung zur Ausweitung der Unfallversicherung auf Berufskrankheiten vom 16. Dezember 1936 hatte die Liste auf 26 erweitert, wobei Pressluftschäden lediglich die neue Nummer 16 erhielten. Eine inhaltliche Veränderung brachte die Vierte Verordnung vom 20. Januar 1943. 
Rückwirkend zum 1. Januar 1942 fielen unter Nr. 16 und 16a nicht nur Schädigungen durch gleichartige Maschinen, Anklopf- und Druckluftmaschinen. Darüber hinaus wurde die Entschädigungspflicht „unter Ausschluß der neurotisch bedingten Krankheitszustände auf alle örtlichen Gewebsund Funktionsstörungen ausgedehnt", wie es im offiziellen Kommentar des Ministerialbeamten Michael Bauer hieß (Bauer 1943: 135).

Knapp 15 Jahre nach der Einschränkung auf Muskeln, Knochen und Gelenke wurden unter den Bedingungen der Kriegswirtschaft nun auch Nerven, Sehnen und Gefäße eingeschlossen. Zahlenmäßig war in erster Linie der Einbezug aller vibrierenden Arbeitsmaschinen relevant, während der Gewerbearzt Erich Beintker von der Ausweitung der entschädigungspflichtigen Erkrankungen keine quantitativen Effekte erwartete, was sich im Nachhinein bestätigte (Hagen 1965: 767, 773-774). „Die Schwierigkeiten bei der Klärung der Zusammenhänge einer Erkrankung mit der Einwirkung der Vibrationen sind nicht geringer geworden“, so Beintker, und weiter: „Wesentlich ist aber, daß der Ablehnungsgrund ,Es liegt eine Erkrankung der Nerven vor" hinfällig geworden ist". ${ }^{18}$

\section{Ausblick}

Die hier ausgelegten Fäden der Begutachtung ergeben einige lose Enden, die unter anderem zur wachsenden Bedeutung von ,arbeitsbezogenen Muskel-Skelett-Erkrankungen“ im späteren 20. Jahrhundert weitergesponnen werden könnten. Was sich in dieser Detailstudie einer neuen Berufskrankheit abzeichnet und weiter verfolgt werden müsste, sind Routinen, die es erlaubten, die Begutachtung der 1929 medizinisch weitgehend unbekannten Pressluftschäden in ein über Jahrzehnte eingespieltes System einzufügen, dessen Entstehung und Transformation, seine Ermöglichungen und Beschränkungen lohnenswerter Gegenstand einer größeren Untersuchung wären. Dabei wäre der Blick auch auf Konkurrenz und Zusammenspiel von unterschiedlichen - seriellen, experimentellen, apparativen, kasuistisch-hermeneutischen und weiteren - Evidenzstrategien zu richten (vgl. etwa Martin 2014: 233-242). Das Beispiel Pressluftschäden lädt insgesamt dazu ein, sich medizinische Begutachtung als strukturierten, aber grundsätzlich offenen Prozess vorzustellen, in dem sich praktisches Wissen Zug um Zug in Interaktionen von Akteuren aus unterschiedlichen Handlungsfeldern wie Gesetzgebung, Medizin, Sozialverwaltung und Justiz formierte, wobei ein vollständiges Bild der Wissensbildung weitere Stimmen stärker einbeziehen müsste - etwa die Versicherten, ihre Rechtsanwälte, 
aber auch Fachleute wie Ingenieure und technische Aufsichtsbeamte im Bergbau, die mit eigenen Gutachten in Erscheinung traten. ${ }^{19}$

Sicherlich gab es Machtgefälle und die Akteure verfügten über ungleiche Ressourcen, sich an der Wissensbildung zu beteiligen. Aber gerade weil es sich beim Bergmannsheil um ein arbeitgebernahes Krankenhaus handelt, das zentralisierte Begutachtung im Auftrag der Berufsgenossenschaft betrieb, zeigt sich deutlich die Eigenlogik der medizinischen Begutachtungspraxis, die neben dem bestellten Wissen auch solches produzierte, das nicht vorgesehen war. Die Routinen der Begutachtung behaupteten sich zumindest teilweise gegenüber den Rationalitäten des Sozialstaats oder den Interessen der Auftraggeber, unter anderem weil sie in einem nicht aufzulösenden Spannungsfeld zwischen medizinisch generalisiertem Wissen und einzelfallbezogenem Entscheidungswissen angesiedelt waren. So agierten weder die Ärzte am Bergmannsheil als Erfüllungsgehilfen von Berufsgenossenschaft oder "Staat", noch lassen sich Paul Rostocks Karriere im Nationalsozialismus und seine Beteiligung an Medizinverbrechen (Schmiedebach \& Schwoch 2001b: 69-73) einfach als Fortsetzung seiner restriktiven sozialrechtlichen Gutachten lesen. Vielmehr fordern sie dazu auf, „soziale Medizin“ in ihren verschiedenen Facetten - als Medizin für die Notleidenden und Benachteiligten, als Medizin im Sozialstaat und als Medizin im Namen der "Gemeinschaft" - historisch neu zu verorten.

\section{Danksagung}

Der Beitrag ist im Kontext der ERC-Forschergruppe „Ways of Writing: How Physicians Know, 1550-1950“ (2012-2017) entstanden, deren Mitgliedern ich für Kritik und Anregungen ebenso danke wie den Gutachter_innen des Peer-review-Verfahrens.

\section{Anmerkungen}

1 Vgl. etwa Ewald (1986); Rabinbach (1992); Sarasin \& Tanner, Hg. (1998); Lengwiler (2006); Bluma (2012).

2 Die entsprechenden Krankenakten sind nach Auskunft des Instituts für Medizinische Ethik und Geschichte der Medizin der Universität Bochum nicht vorhanden bzw. erschlossen.

3 Entscheidungen und Mitteilungen des Reichsversicherungsamts, 30, 1931, 7 (Bk 957/30). 
4 Aktenvermerk über die Besprechung im Reichsarbeitsministerium, 22.10.1928, R 89/ 15130, p. 287r. Sämtliche in diesem Aufsatz zitierte Archivalien stammen aus dem Bundesarchiv, Bestand R 89 (Reichsversicherungsamt).

5 Aktenvermerk: Protokoll Tagung der Gewerbeärzte im RAM, 11.12.1930, R 89/15131, p. $110 \mathrm{r}$.

6 Die Berufsgenossenschaft, 49, 1934, 142 (Ia 5131/32/12). Vgl. etwa R 89/23003: Bk 369/29 Wilhelm S. gg. Knappschafts-BG, p. 7v; Bk. 424/29 Josef S. gg. KnappschaftsBG, p. 9v.; Bk 467/29 Wilhelm T. gg. Knappschafts-BG, p. 7v.

7 Aktennotiz 26.11.[1932], R 89/23026, Bk 218/1931, Heinrich K. gg. Knappschafts-BG, p. 34v.

8 Gutachten Dr. Zumstein, 25.07.1932, R 89/23026, Bk 218/1931 Heinrich K. gg. Knappschafts-BG, p. 20r-21v.

9 Vgl. etwa R 89/23015, Bk 1052/1930 Kaspar H. gg. Reichsbahn-Direktion Köln, p. 20-23; R 89/23026, Bk 387/1931 Fritz K. gg. Knappschafts-BG, p. 18-23; R 89/23015, Bk 754/1930 Gustav B. gg. Reichsbahn-Direktion Köln; R 89/23003, Bk 469/29 August R. gg. Knappschafts-BG, p. 26.

10 Vgl. R 89/23001, Bk 100/29 Fabian P. gg. Knappschafts-BG, p. 15-31; R 89/23013, Bk 1752/29 Karl R. gg. Knappschafts-BG, p. 12-22; R 89/23003, Bk 469/29 August R. gg. Knappschafts-BG, Pressluft, p. 22-38; R 89/23017, Bk 1843/1930 Anton K. gg. Knappschafts-BG, p. $42-48$.

11 R 89/23018, Bk 2360/1930 Johann S. gg. Steinbruchs-BG.

12 R 89/23003, Bk 388/29 Wilhelm S. gg. Knappschafts-BG, p. 10v, 13r; vgl. auch Bericht und Gutachten Schwartz, 09.04.(1931) R 89/23017, Bk 1843/1930 Anton K. gg. Knappschafts-BG, p. 55r-v.

13 Aktennotiz 16.06.[1930], R 89/23001, Bk 100/29 Fabian P. gg. Knappschafts-BG, p. 62v.

14 Vgl. die Kursprogramme in: Kompaß, 50, 1935, 41; Kompaß 52, 1937, 32.

15 Entscheidungen und Mitteilungen des Reichsversicherungsamts, 30, 1931, 6-7 (Bk 957/30).

16 45. Rundschreiben an die Herren Knappschafts-Bezirks- und Fachärzte, 23.10.1931. Die Knappschaft, 7, 1931, 359-360.

17 Die Berufsgenossenschaft, 49, 1934, 11 (Ia 10913/31/2).

18 Niederschrift über die wissenschaftliche Tagung der Staatlichen Gewerbeärzte in Dresden am 29. und 30. Juni 1943 [...], R 89/15139, p. 162v-163r.

19 Vgl. R 89/23001, Bk 100/29 Fabian P. gg. Knappschafts-BG, p. 45-47; R 89/23026, Bk 542/1931 Ernst H. gg. Nordwestliche Eisen- und Stahl-BG, p. 28-30.

\section{Literatur}

Andreesen, Remmer 1934a. Das reizempfindliche Knie. Unter besonderer Berücksichtigung der Meniscusschäden der Bergleute. Archiv für orthopädische und Unfall-Chirurgie (35): 58-72.

Andreesen, Remmer 1934b. Meniscusschaden, Unfallfolge oder Berufserkrankung? Zentralblatt für Chirurgie (61): 1235-1239.

Andreesen, Remmer 1937a. Gemeinsame Anschauungen in der Beurteilung der Meniscusschäden. Zentralblatt für Chirurgie (64): 222-228.

Andreesen, Remmer 1937b. Meniscusbeschädigungen (Verletzungen und Erkrankungen) bei Sport und Arbeit. Ergebnisse der Chirurgie und Orthopädie (30): 24-128.

Andreesen, Remmer 1942a. Über Verschlimmerung von „Preßluftschäden“. Archiv für orthopädische und Unfall-Chirurgie (42): 121-129. 
Andreesen, Remmer 1942b. Gelenkschäden durch Preßluftarbeit. Medizinische Klinik (38): 200-202.

Anonymus 1926. Gesundheitsschädigungen durch Preßluftwerkzeuge. Gewerkschafts-Zeitung (36): 671-672.

Anonymus 1938. Die Voraussetzungen für die Entschädigung von Meniskusschäden. Komраß (53): 81-82.

Barta, Heinz 1983. Kausalität im Sozialrecht. Entstehung und Funktion der sogenannten Theorie der wesentlichen Bedingung. 2 Bände, Berlin: Duncker \& Humblot.

Bauer, Michael 1943. Die Vierte Verordnung über Ausdehnung der Unfallversicherung auf Berufskrankheiten. Reichsarbeitsblatt Nr. 9, II: 133-138.

Bauer, Michael J. und Hermann Gerbis 1931. Entschädigungspflichtige Berufskrankheiten. In: Hans Liniger, Raphael Weichbrodt und Albrecht Wilhelm Fischer (Hg.). Handbuch der ärztlichen Begutachtung. Bd. 1, Leipzig: Barth: 207-259.

Beintker, Erich 1930. Über „Muskelatrophie“ durch Preßluftwerkzeuge. Archiv für Gewerbepathologie und Gewerbehygiene (1): 376-379.

Bluma, Lars 2010. Heterotope Orte: Raumhistorische Dimensionen des knappschaftlichen Krankenhauswesens im Ruhrgebiet. In: Christoph Bartels (Hg.). Berufliches Risiko und soziale Sicherheit. Beiträge zur Tagung „Vergangenheit und Zukunft sozialer Sicherungssysteme am Beispiel der Bundesknappschaft und ihrer Nachfolger" im Deutschen Bergbau-Museum Bochum, 8. und 9. Oktober 2009. Bochum: DBM: 67-98.

Bluma, Lars 2012. Der Körper des Bergmanns in der Industrialisierung. Biopolitik im Ruhrkohlenbergbau 1890-1980. In: Lars Bluma und Karsten Uhl (Hg.). Kontrollierte Arbeit - disziplinierte Körper? Zur Sozial- und Kulturgeschichte der Industriearbeit im 19. und 20. Jahrhundert. Bielefeld: Transcript: 35-72.

Bluma, Lars 2015. Die Objektivierung des bergmännischen Körpers. Praktiken der Sichtbarmachung im Kontext von Versicherungsrationalität und berufsspezifischen Krankheiten. In: Sybilla Nikolow (Hg.). Erkenne Dich selbst! Strategien der Sichtbarmachung des Körpers im 20. Jahrhundert. Köln: Böhlau: 269-285.

Boyer, Josef 1990. Bergmannsheil 1890-1990. In: Bergbau-Berufsgenossenschaft, Bochum (Hg.). 100 Jahre Bergmannsheil. Gütersloh: o.V.: 37-83.

Boyer, Josef 1995. Unfallversicherung und Unternehmer im Bergbau. Die Knappschafts-Berufsgenossenschaft 1885-1945. München: Beck.

Burghardt, Uwe 1995. Die Mechanisierung des Ruhrbergbaus 1890-1930. München: Beck.

Bürkle de la Camp, Heinrich 1937. Über die Erkrankung der Muskeln, Knochen und Gelenke durch Arbeiten mit Preßluftwerkzeugen. Die Medizinische Welt (11): 1348-1351.

Bürkle de la Camp, Heinrich 1938. Die Berufskrankheit Nr. 16. „Erkrankungen der Muskeln, Knochen und Gelenke durch Arbeiten mit Preßluftwerkzeugen“. Zeitschrift für ärztliche Fortbildung (35): 485-489.

Bürkle de la Camp, Heinrich 1939a. Neuere Erkenntnisse in der Beurteilung der Gewebsschädigungen durch Arbeiten mit Preßluftwerkzeugen. Archiv für orthopädische und Unfall-Chirurgie (40): 161-170.

Bürkle de la Camp, Heinrich 1939b. Allgemeine Knochen- und Gelenkschäden einschließlich Arthritis deformans. In: Albrecht Wilhelm Fischer und Gustav Molineus (Hg.). Das ärztliche Gutachten im Versicherungswesen. Bd. 1, Leipzig: Barth: 48-96.

Büro des vorläufigen Reichswirtschaftsrats (Hg.) 1933. Der vorläufige Reichswirtschaftsrat 1927-1932. Berlin: Reichsverlagsamt.

Dembe, Allard E. 1996. Occupation and Disease: How Social Factors Affect the Conception of Work-Related Disorders. New Haven: Yale University Press.

Ewald, François 1986. L'état providence. Paris: Grasset.

Geisthövel, Alexa und Volker Hess 2017. Handelndes Wissen. Die Praxis des Gutachtens. In: Alexa Geisthövel und Volker Hess (Hg.). Medizinisches Gutachten. Geschichte einer neuzeitlichen Praxis. Göttingen: Wallstein: 9-39.

Hagen, Josef 1965. Klinik der entschädigungspflichtigen Berufskrankheiten. In: Heinrich Bürkle de la Camp und Max Schwaiger (Hg.). Handbuch der gesamten Unfallheilkunde. 3. Aufl. Stuttgart: Enke, Bd. 3: 601-874.

Hamilton, Alice 1930. A Vasomotoric Disturbance in the Fingers of Stonecutters. Archiv für Gewerbepathologie (1): 348-358. 
Hebestreit, Hermann und Heinrich Bartsch 1937. Die Berufskrankheiten in der Unfallversicherung auf Grund der Dritten Verordnung über Ausdehnung der Unfallversicherung auf Berufskrankheiten vom 16. Dezember 1936 [...]. Berlin: Langewort.

Hennig, Otto und Alfred Gallinek 1933. Schultermuskellähmung, hervorgerufen durch Arbeit mit Preßluftbohrhammer. Archiv für orthopädische und Unfall-Chirurgie (34): $149-154$.

Hohmann, Joachim S. 1997. Berufskrankheiten im Prozeß der Modernisierung. Eine sozialhistorische und soziologische Studie. Frankfurt am Main: Peter Lang.

Holtzmann, Friedrich 1926. Eine Gelenkveränderung durch Arbeit mit Presslufthämmern. Zentralblatt für Gewerbehygiene (3): 235-237.

Holtzmann, Friedrich 1930. Erkrankungen der Muskeln, Knochen und Gelenke durch Arbeiten mit Preßluftwerkzeugen. In: Fabrikärzte der chem. Industrie (Hg.). Ärztliche Merkblätter über berufliche Erkrankungen [...]. 3. Aufl. Berlin: Springer: 69-71.

Junghanns, Herbert 1936. Gefäßschädigung durch Arbeit mit Preßluftwerkzeug. Archiv für orthopädische und Unfall-Chirurgie (37): 421-423.

Kelzenberg, Fritz 1941. Bericht über die Nachuntersuchung von 150 Arbeitern, die 1929-1934 erstmalig wegen einer Schädigung durch Arbeit mit Pressluftwerkzeugen begutachtet wurden. Diss. Med. Universität München.

Knoll-Jung, Sebastian 2017. Zwischen Verfahrenslogik und ärztlichem Eigensinn. Die Praxis ärztlicher Begutachtung in der Unfallversicherung 1884-1914. In: Alexa Geisthövel und Volker Hess (Hg.). Medizinisches Gutachten. Geschichte einer neuzeitlichen Praxis. Göttingen: Wallstein: 70-92.

[Koelsch, Franz] 1929. Nr. 14: Erkrankungen der Muskeln, Knochen und Gelenke durch Arbeiten mit Preßluftwerkzeugen. In: Die Ausdehnung der Unfallversicherung auf Berufskrankheiten. Zweite Verordnung des Reichsarbeitsministers vom 11. Februar 1929. Erläutert von M. Bauer, H. Engel, F. Koelsch und J. Krohn. Berlin: Hobbing: 222-224.

Köstler, Josef 1935. Anatomische Beobachtungen zur Frage der Entstehung des Mondbeintodes. Archiv für orthopädische und Unfall-Chirurgie (36): 34-40.

Laarmann, Alois 1936. Bericht über den Verhandlungsgegenstand Meniskusschäden in der Sitzung am 18. Sept. nachm. Röntgenpraxis (8): 772-776.

Laarmann, Alois 1940. Beitrag zu „Neuere Erkenntnisse in der Beurteilung der Gewebsschädigungen durch Arbeiten mit Preßluftwerkzeugen“. Monatsschrift für Unfallheilkunde (47): 65-71.

Laarmann, Alois 1944. Der Preßluftschaden. Leipzig: Thieme.

Lammel, Hans-Uwe 1994. Chirurgie und Nationalsozialismus am Beispiel der Berliner Chirurgischen Universitätsklinik in der Ziegelstraße. In: Wolfram Fischer et al. (Hg.). Exodus von Wissenschaften aus Berlin. Fragestellungen - Ergebnisse - Desiderate. Entwicklungen vor und nach 1933. Berlin: de Gruyter: 568-591.

Leitfaden zur Dritten Verordnung über Ausdehnung der Unfallversicherung auf Berufskrankheiten vom 16. Dezember 1936 (RGBl I S. 1117). Herausgegeben von der Sektion II der Knappschafts-Berufsgenossenschaft, Bochum, unter Mitwirkung der Sektion IV der Berufsgenossenschaft der chemischen Industrie, Köln. Bochum 1937.

Leitfaden zur Zweiten Verordnung über Ausdehnung der Unfallversicherung auf Berufskrankheiten vom 11. Februar 1929 (RGBl. I Seite 27) enthaltend eine Sammlung von Entscheidungen der Spruchbehörden [...]. Für Berufsgenossenschaften, Krankenkassen, Kreis-, Gewerbe- und andere Ärzte, Gewerbeaufsichtsbeamte, Versicherungs- und Oberversicherungsämter, Landesversicherungsanstalten, Krankenhäuser usw. Hg. von der Sektion 2 der Knappschafts-Berufsgenossenschaft, Bochum, [...]. Bochum 1934.

Lengwiler, Martin 2006. Risikopolitik im Sozialstaat. Die schweizerische Unfallversicherung 1870-1970. Köln: Böhlau.

Lengwiler, Martin 2009. Internationale Expertennetzwerke und nationale Sozialstaatsgeschichte: Versicherung der Silikose in Deutschland und der Schweiz (1900-1945). Journal of Modern European History (7): 197-218.

Lengwiler, Martin 2017. Standardisierung oder Individualdiagnostik? Ärztliche Gutachten im Versicherungswesen des 20. Jahrhunderts. In: Alexa Geisthövel und Volker Hess (Hg.). Medizinisches Gutachten. Geschichte einer neuzeitlichen Praxis. Göttingen: Wallstein: 95-120. 
Linde, Fritz 1935. Zur Frage der Entstehung des Bandscheibenrisses. Medizinische Klinik (31): 942-943.

[Lipmann, Otto] 1928. Die Arbeitsverhältnisse im Steinkohlenbergbau in den Jahren 19121926 dargest. auf Grund der dem Arbeitsleistungsausschu $\beta$ vorliegenden Materialien und Verhandlungsergebnisse. Berlin: Mittler.

Lohmar, Paul 1929. Unfallmedizinische Arbeitsgemeinschaften. Die Berufsgenossenschaft (44): 549-560.

[Loriga, Giovanni] 1930. Pneumatic Tools. In: International Labor Office (Hg.). Occupation and Health. Encyclopedia of Hygiene, Pathology and Social Welfare. Bd. 2, Genf: o.V., No 162.

Magnus, Georg 1939. [Diskussionsbeitrag]. In: Bericht über den VIII. Internationalen Kongress für Unfallmedizin und Berufskrankheiten Frankfurt a. M. 26. bis 30. September 1938. Bd. II, Leipzig: Thieme: 361.

Martin, Michael 2002. Staublungenerkrankungen in der Geschichte des Bergbaus. In: Detlev Jung und Klaus-Dieter Thomann (Hg.). Berufskrankheitenrecht. Beiträge zur Geschichte und Gegenwart der Berufskrankheiten und des Berufskrankheitenrechts [Interdisziplinäres Symposium zur Geschichte und Gegenwart der Berufskrankheiten und des Berufskrankheitenrechts, Mainz, 9./10. Februar 2001]. Stuttgart: Gentner-Verlag: 101-115.

Martin, Michael 2014. Spuren der Arbeit. Zur Beweiskraft des Röntgenbildes bei der Anerkennung der Silikose als Berufskrankheit. Westfälische Forschungen (64): 1-20.

Milles, Dietrich 1999. Ludwig Teleky und die Soziale Medizin. In: Dietrich Milles und Norbert Schmacke (Hg.). Ludwig Teleky und die Westdeutsche Sozialhygienische Akademie. Arbeiten für eine soziale Medizin (1903-1939). Düsseldorf: Akademie für öffentliches Gesundheitswesen: 9-54.

Moses, Julia 2012. Contesting Risk: Specialist Knowledge and Workplace Accidents in Britain, Germany, and Italy, 1870-1920. In: Kerstin Brückweh, Richard Wetzell, Benjamin Ziemann und Dirk Schumann (Hg.). Engineering Society. The Role of the Human and Social Sciences in Modern Societies, 1880-1980. Basingstoke: Palgrave Macmillan: 50-78.

Ostermann, Wolfgang 1976. Die Geschichte der „Berufsgenossenschaftlichen Krankenanstalten Bergmannsheil Bochum“. Münster: Murken-Altrogge.

Rabinbach, Anson 1992. The Human Motor. Energy, Fatigue, and the Origins of Modernity. Berkeley u. a.: University of California Press.

Rainhorn, Judith 2014. Observer les corps, délier les langues, ou comment dévoiler les interactions entre travail et santé dans les mines de cuivre de l'Arizona (États-Unis, 1919). In: Judith Rainhorn (Hg.). Santé et travail à la mine, XIXe-XXIe siècle. Villeneuve d'Ascq: Septentrion: 267-286.

Raphael, Lutz 1996: Die Verwissenschaftlichung des Sozialen als methodische und konzeptionelle Herausforderung für eine Sozialgeschichte des 20. Jahrhunderts. Geschichte und Gesellschaft (22): 165-193.

Rosental, Paul-André (Hg.) 2009. Health and Safety at Work in a Transnational Perspective. Journal of Modern European History (7). ISSN 1611-8944

Rostock, Paul 1930. Durch Arbeit mit Preßluftwerkzeugen hervorgerufene Veränderungen am Ellenbogengelenk. In: Verhandlungen der Deutschen Gesellschaft für Chirurgie. 54. Tagung. 23.-26. April 1930. Berlin: Springer: 69-71.

Rostock, Paul 1931a. Durch Arbeit mit Preßluftwerkzeugen hervorgerufene Veränderungen am Ellenbogengelenk. Archiv für orthopädische und Unfall-Chirurgie (29): 285-290.

Rostock, Paul 1931b. Die Zusammenhänge zwischen der Dauer von Preßluftwerkzeugarbeit und Gelenkschäden. Monatsschrift für Unfallheilkunde (38): 337-349.

Rostock, Paul 1933. Erkrankungen der Muskeln, Knochen und Gelenke durch Arbeit mit Preßluftwerkzeugen. In: Fritz König und Georg Magnus (Hg.). Handbuch der gesamten Unfallheilkunde. Bd. 2, Stuttgart: Enke: 89-111.

Rostock, Paul 1934a. Die Zunahme einer Ellenbogengelenkschädigung durch Arbeit mit Preßluftwerkzeugen bei Fortsetzung dieser Arbeit. Monatsschrift für Unfallheilkunde (41): 43-45.

Rostock, Paul 1934b. Gelenkschäden durch chronische Erschütterungen. Zentralblatt für Chirurgie (61): 630-634.

Rostock, Paul 1935a. Unfallbegutachtung. Leipzig: Barth. 
Rostock, Paul 1935b. Reaktion des Ellenbogengelenks auf abnorm starke Arbeitsbeanspruchung. Archiv für orthopädische und Unfall-Chirurgie (36): 502-504.

Rostock, Paul 1936a. Formveränderungen des menschlichen Ellenbogengelenks durch abnorm starke Arbeitsbeanspruchung. Röntgenpraxis (8): 303-306.

Rostock, Paul 1936b. Gelenkschäden durch Arbeit mit Preßluftwerkzeugen und andere schwere körperliche Arbeit. Medizinische Klinik (32): 341-343.

Sarasin, Philipp und Jakob Tanner (Hg.) 1998. Physiologie und industrielle Gesellschaft. Studien zur Verwissenschaftlichung des Körpers im 19. und 20. Jahrhundert. Frankfurt a. M.: Suhrkamp.

Scheffler, Hans 1927. Beobachtungen und Ergebnisse bei einer fünfï̈hrigen Frakturenbehandlung. Berlin: Springer.

Schlich, Thomas 2006. Trauma Surgery and Traffic Policy in Germany in the 1930s: A Case Study in the Co-Evolution of Modern Surgery and Society. Bulletin for the History of Medicine (90): 73-94.

Schmiedebach, Heinz Peter und Rebecca Schwoch 2001a. Prof. Dr. med. Richard Hugo Georg Magnus. In: Michael Sachs, Heinz-Peter Schmiedebach und Rebecca Schwoch (Hg.). Deutsche Gesellschaft für Chirurgie 1933-1945. Die Präsidenten. Heidelberg: Kaden: 33-62.

Schmiedebach, Heinz Peter und Rebecca Schwoch 2001b. Exkurs. In: Michael Sachs, HeinzPeter Schmiedebach und Rebecca Schwoch (Hg.). Deutsche Gesellschaft für Chirurgie 1933-1945. Die Präsidenten. Heidelberg: Kaden: 63-73.

Schramm, Hans 1938. Erkrankungen der Muskeln, Knochen und Gelenke durch Arbeit mit Preßluftwerkzeugen in der Eisen- und Stahlindustrie. Archiv für orthopädische und Unfall-Chirurgie (39): 248-257.

Seyring, Maria 1930. Erkrankungen durch Arbeit mit Preßluftwerkzeugen. Archiv für Gewerbepathologie (1): 359-375.

Shin, Myoung-Hoon 2007. Die Arbeitersekretariate in der deutschen Arbeiterbewegung. Eine Institution für die gewerkschaftliche Sozialpolitik in Wechselbeziehungen zur staatlichen Sozialpolitik im Kaiserreich, im Ersten Weltkrieg und in der Weimarer Republik. Lübeck/Marburg: Der Andere Verlag.

Sniegs, Monika 1993. Quantitative Analyse der Berufskrankheitenverfahren in der Unfallversicherung 1925-1933 In: Dietrich Milles (Hg.). Gesundheitsrisiken. Industriegesellschaft, und soziale Sicherungen in der Geschichte. Bremerhaven: Verlag für neue Wissenschaft: 191-218.

Stockhausen, Heinz-Peter 1967. Zur Bedeutung und versicherungsrechtlichen Beurteilung der Berufskrankheit Nr. 25 der Anlage zur VI. Berufskrankheitenverordnung (Preßluftschädigung). Kompaß (77): 181-188.

Thomann, Klaus Dieter 1993. „Ich habe mir die Gesundheit ruiniert ... “ Preßlufterkrankungen der Gelenke und die Anfänge der Berufskrankheitenverordnung 1929 bis 1936. In: Dietrich Milles (Hg.). Gesundheitsrisiken. Industriegesellschaft, und soziale Sicherungen in der Geschichte. Bremerhaven: Verlag für neue Wissenschaft: 317-349.

Weber, F. W. A. 1934. Über Schädigung des Nervus ulnaris durch Arbeit mit Preßluftwerkzeugen. Monatsschrift für Unfallheilkunde (41): 75-81.

Wette, Walter 1931. Die Lunatumnekrose als Unfallfolge und Berufskrankheit. Archiv für orthopädische und Unfall-Chirurgie (29): 299-319.

\author{
Alexa Geisthövel \\ Institut für Geschichte der Medizin und Ethik in der Medizin \\ Charité - Universitätsmedizin Berlin \\ Thielallee 71 \\ 14195 Berlin \\ Deutschland \\ alexandra.geisthoevel@charite.de
}

